\title{
Foucault statt Fürst? Gedanken zu einem an Bedeutung gewinnenden Paradigma
}

\author{
Gerd Lintz ${ }^{1}$
}

Eingegangen: 21. Oktober 2016 / Angenommen: 26. April 2017 / Online publiziert: 8. Mai 2017

(C) Springer-Verlag Berlin Heidelberg 2017

Zusammenfassung Vor allem bezugnehmend auf die Beiträge von Zimmermann (2012) sowie Balke und Reimer (2016) in der Zeitschrift ,Raumforschung und Raumordnung" setzt sich der Zwischenruf mit den wissenschaftlichen Arbeiten auseinander, die unter den Stichworten Konstruktivismus, Diskursanalyse und Gouvernementalitätsforschung immer größere Verbreitung finden. Der Autor schildert am Beispiel der beiden genannten Beiträge seine Gedanken zum Verhältnis des aufstrebenden sozialkonstruktivistischen Paradigmas zum (bisherigen) Mainstream. Er plädiert dafür, in einer offenen Diskussion das Verständnis für die Gemeinsamkeiten und Unterschiede der Ansätze zu verbessern.

Schlüsselwörter Konstruktivismus · Diskurs · Gouvernementalität · Landschaft · Region

\section{Foucault instead of Fürst? Thoughts on an increasingly important paradigm}

\begin{abstract}
Especially drawing on the contributions of Zimmermann (2012) and Balke and Reimer (2016) published in the journal "Raumforschung und Raumordnung", this article deals with research done under the keywords constructivism, discourse and governmentality. Taking the example of those two contributions the author presents his thoughts on the relationship between the emerging social constructivist paradigm on the one hand and the (previous) main-
\end{abstract}

Dr. Gerd Lintz

g.lintz@ioer.de

1 Leibniz-Institut für ökologische Raumentwicklung, Weberplatz 1, 01217 Dresden, Deutschland stream on the other hand. He argues for an open discussion in order to improve the understanding of commonalities and divergences between these approaches.

Keywords Constructivism · Discourse - Governmentality · Landscape $\cdot$ Region

\section{Ein an Bedeutung gewinnendes Paradigma in der Raumwissenschaft}

Explizit sozialkonstruktivistische Forschungsansätze wie die Diskursanalyse sind bislang in den Raumwissenschaften noch nicht (völlig) etabliert. ,Gleichwohl erfreuen sie sich aber gerade auch bei vielen jüngeren Wissenschaftlern einer wachsenden Beliebtheit" (Mattissek/Pfaffenbach/ Reuber 2013: 293). Vielen deutschen Raumwissenschaftlern und Praktikern begegnet deshalb immer öfter ein gänzlich neues Vokabular, das in der internationalen Literatur schon häufiger zu finden ist: Phänomene werden konstituiert, produziert und reproduziert oder konstruiert, rekonstruiert und dekonstruiert. Es geht zum Beispiel um Praktiken, hegemoniale Diskurse, Kodierung, Poststrukturalismus und Gouvernementalität, wobei der Name Michel Foucault vielfach genannt wird.

Hinter diesem Vokabular stehen vom bisherigen Mainstream zum Teil erheblich abweichende wissenschaftstheoretische Konzepte und inhaltliche Forschungsschwerpunkte. Es überrascht deshalb nicht unbedingt, dass das Verständnis der Vertreter der verschiedenen wissenschaftlichen Lager füreinander an Grenzen stößt. Auch Praktiker tun sich oft mit dem neuen Ansatz schwer. Ziel dieses Diskussionsbeitrages ist es deshalb, am Beispiel von zwei in der Zeitschrift „,Raumforschung und Raumordnung“ veröffentlichten Beiträgen das zu spürende Unbehagen auf al- 
len Seiten aufzuzeigen und einige Gedanken zum Verhältnis des aufstrebenden Paradigmas zum (bisherigen) Mainstream zu formulieren. Dies erfordert eine Auseinandersetzung mit den unübersichtlichen Feldern der Methodologie empirischer Sozialforschung und der sozialwissenschaftlichen Theorieansätze.

\section{Eine Reaktion: Unverständnis für die Landschaftsforschung}

Horst Zimmermann (2012: 481) hatte in der Rubrik ,Zur Diskussion“ dieser Zeitschrift auf die Aufsätze im Schwerpunktheft 2/2012, „Die gesellschaftliche Konstituierung von Kulturlandschaft" geantwortet, dass diese für einen Ökonomen nicht nachvollziehbar seien. Das Schwerpunktheft beschäftigte sich aus sozialkonstruktivistischer Perspektive mit der Frage, ,wie sich Kulturlandschaften erst durch das Zusammenwirken ganz unterschiedlicher Institutionensysteme und Akteure im politischen Handeln, durch deren Raumbilder und Diskurse konstituieren“ (Kilper/Heiland/ Leibenath et al. 2012: 92).

Zimmermann (2012) präsentiert demgegenüber ,nüchtern" die Perspektive der ökonomischen Lehre, wonach Landschaft als regionales öffentliches Gut zu verstehen ist, das bei entsprechender Wertschätzung durch die Wirtschaftssubjekte (Nutzung ökonomischer Bewertungsmethoden) mithilfe der öffentlichen Politik zu sichern bzw. zu gestalten ist. Diese Analyse ist anerkannt und ohne Zweifel hilfreich. Was die Ökonomen (ausgehend von ihrem Blick auf einzelne als völlig souverän angesehene Individuen) aber üblicherweise nicht thematisieren, sind - unter anderem - die berechtigten und viel grundsätzlicheren gesellschaftswissenschaftlichen Fragen, woher die Vorstellung, was Landschaft ist, und der Grad der Wertschätzung dafür überhaupt kommen. Das genau gehörte jedoch zum Forschungsgegenstand derer, die das Schwerpunktheft „Die gesellschaftliche Konstituierung von Kulturlandschaft" gestalteten. Für viele Ökonomen - und letztlich Vertreter anderer Disziplinen - ist die Landschaft einfach da und ihre Wertschätzung muss nur mit bestimmten Methoden gemessen und sie dann entsprechend gestaltet werden. Um verstehen zu können, wie sich die Perspektiven so stark unterscheiden können, muss man etwas weiter ausholen.

\section{Ein Hintergrund: der Kritische Rationalismus und seine Grenzen}

Im Bereich der Sozialwissenschaften sind es generell die Ökonomen, die aus wissenschaftstheoretischer Sicht am konsequentesten dem in Deutschland vielbeachteten und insbesondere mit dem Namen Karl Poppers verbundenen
Kritischen Rationalismus (vgl. Prim/Tilmann 2000 oder Mattissek/Pfaffenbach/Reuber 2013: 43 ff.) folgen und dadurch auch Gefahr laufen, bestimmte Fragestellungen aus den Augen zu verlieren. Der Kritische Rationalismus ist eines von mehreren Konzepten, die aufzeigen, wie die Wissenschaft Wissen über die Welt schaffen soll, und dabei auch ein bestimmtes Wesen des Untersuchungsgegenstandes unterstellen. Er stammt aus den Naturwissenschaften und setzt das Ziel, auf der Basis von empirischen Beobachtungen regelhafte Zusammenhänge in Form von allgemeinen Gesetzen über die Welt zu formulieren. Phänomene sollen erklärt werden anhand der Logik von Experimenten, wobei die abhängigen und unabhängigen Variablen und ihr Verhältnis zueinander genau zu quantifizieren sind. Popper stellt dabei im Gegensatz zum Positivismus, auf dem er ansonsten aufbaut, heraus, dass Erklärungen nie endgültig als wahr oder objektiv angesehen werden können. Theorien gelten immer nur als vorläufig bestätigt und müssen sich in Tests gegenüber Falsifizierungsversuchen behaupten (Popper 1994 [1935]). So nähert sich die Wissenschaft Stück für Stück an die Welt und die Zusammenhänge in ihr an, wobei diese aber letztlich als objektiv vorhanden und als unabhängig von sozialen Akteuren angesehen werden (Objektivismus; Bryman 2004: 16).

Dem hohen Anspruch des Kritischen Rationalismus kann übrigens die praktische Raumwissenschaft häufig gar nicht gerecht werden. Im Falle einer methodologisch rigorosen Beurteilung im Sinne des Kritischen Rationalismus und Poppers würden hier viele übliche Studien überhaupt nicht als wissenschaftlich anerkannt werden. $\mathrm{Zu}$ denken ist etwa an die Entwicklung von Methoden, die Gestaltung von Instrumenten zur Erreichung politischer Ziele, Evaluationen, die den Einsatz von politischen Maßnahmen untersuchen, alle qualitativen Studien, in denen Variablen nicht quantifiziert sind, sowie explorative Fallstudien, die allenfalls dem vorwissenschaftlichen Bereich zugeordnet würden. Völlig undenkbar erscheint die Begleitforschung zu Modellprojekten, in denen die Wissenschaftler ihre Distanz zum Forschungsobjekt aufgeben und es sogar zu verändern versuchen.

Überhaupt wird die Übertragung naturwissenschaftlicher Prinzipien auf die Sozialwissenschaft, die eine große Rolle in den Raumwissenschaften spielt, vielfach kritisiert (z. B. Schülein/Reitze 2012: 184 f.). So sind etwa echte Experimente mit Menschen, z. B. Regionalplanern und Stadtverwaltungen, nicht vertretbar und die Kontexte als häufig unübersehbares Dickicht von Einflussfaktoren lassen sich nicht wie im Labor einfach abtrennen. Die Prüfung einzelner Hypothesen ergibt dann nicht immer einen Sinn. Auch beispielsweise die Untersuchung von Identitäten von Menschen entzieht sich häufig einem einfachen Ursache-Wirkungs-Denken. Hier wird dann oft eher von Interpretieren und Verstehen als von Erklären gesprochen. Das Beispiel 
der Identitäten zeigt zugleich, dass soziale Phänomene nicht einfach da sind, sondern stark gesellschaftlich beeinflusst sein können. Landschaft im naturwissenschaftlichen Sinne mag vielleicht noch als ,objektiv “ existent betrachtet werden. Dies dürfte bei der Frage, was soziale Akteure unter Landschaft verstehen und was sie ihnen bedeutet, kaum möglich sein. Die Landschaft existiert bzw. die Bilder davon existieren dann nur in den Köpfen der Betrachter.

Zum Kritischen Rationalismus haben sich folgerichtig viele Alternativen ausgebildet, die sich an unterschiedlichen Aspekten der Kritik festmachen. Hier spielt das Gegenmodell zum Objektivismus, der Konstruktivismus, eine große Rolle, mit dem unter anderem die Diskursanalyse eng verbunden ist.

\section{Eine Alternative: der Konstruktivismus}

Schon Kant hat herausgestellt, dass die Wahrnehmung der Welt über die Sinne erfolgt, die uns kein exaktes Bild der Welt , an sich ' bieten (Sander 2014: 78). Insofern konstruiert unsere biologisch geprägte Wahrnehmung nur eine Vorstellung dessen, was existieren mag. Noch wichtiger ist die soziale Dimension der Konstruktion. Jedes Individuum entwickelt beispielsweise entsprechend seiner Sozialisation eigene kognitive und normative Vorstellungen über die Welt. Zum Teil sind diese Vorstellungen aber - so eine soziologische Grunderkenntnis - insgesamt oder gruppenweise sehr ähnlich, da sie in komplexen und häufig historischlangfristigen gesellschaftlichen Prozessen geprägt werden. Dies spricht im Übrigen gegen eine völlige Souveränität der Subjekte, da zumindest zum Teil von der Gesellschaft explizit und implizit vorgegeben ist, was zur Wahl steht oder was die Subjekte wollen können.

Interessant ist auch das Verhältnis von Wissenschaft und ihrem jeweiligen Untersuchungsgegenstand aus konstruktivistischer Sicht. Es besteht die Auffassung, dass letztlich auch die Wissenschaften mit ihren Denk- und Vorgehensweisen den Untersuchungsgegenstand erst konstituieren bzw. selbst schaffen (Mattissek/Pfaffenbach/Reuber 2013: 262). Auf der Basis dieser (insgesamt) konstruierten Wahrnehmung entwickelt sich schließlich auch die Gesellschaft: Teils wird sie bewusst gestaltet, teils entziehen sich Entwicklungen einer bewussten Steuerung. In beiden Fällen kann ebenfalls von Konstruktion gesprochen werden. Im Entwicklungsprozess verändern sich wiederum auch die - konstruierten - Vorstellungen von Gesellschaft. Es gibt, sozusagen, nur noch abhängige Variablen.

Die Vorstellungen, die in der Gesellschaft und Wissenschaft zu finden sind, und die Gesellschaft selbst können also in vielfältiger Weise als konstruiert verstanden werden. Es lässt sich hier kaum widersprechen. Der entscheidende Punkt ist allerdings, was Wissenschaftler daraus folgern im
Hinblick auf Ziele, Gegenstände und Methoden der Forschung.

Auf der einen Seite lässt sich die Konstruiertheit schon als eine Einschränkung verstehen, die jedoch nicht dazu führt, die Idee der Annäherung an eine objektiv existierende Wirklichkeit aufzugeben. Auch Popper war sich offensichtlich einer gewissen Konstruiertheit der Welt bewusst. Er selbst unterscheidet drei Sphären: die physikalische Welt, das Bewusstsein des Menschen und das Reich der Gedanken, also der Vorstellungen, die sich Menschen von der Welt machen (Schülein/Reitze 2012: 160 f.). In seiner ,Scheinwerfertheorie“ argumentiert er, dass Wissenschaftler immer nur den von ihnen selbst aktiv beleuchteten Teil der Wirklichkeit betrachten (Popper 1984 [1972]: 354 ff.).

Auf der anderen Seite ist darauf hinzuweisen, dass sich auch der Wissenschaftler nicht dem gesellschaftlichen Einfluss entziehen kann. Unter anderem deshalb fragen die Vertreter des Konstruktivismus, auf welche Weise die Welt sozial konstruiert wird und welche Rolle die Konstruktionen spielen (Mattissek/Pfaffenbach/Reuber 2013: 31). Genau hier ist das Schwerpunktheft 2/2012 von „Raumforschung und Raumordnung“ zur gesellschaftlichen Konstituierung von Kulturlandschaft einzuordnen. Wird der Konstruiertheit von sozialen Phänomenen erst einmal eine entscheidende Bedeutung zugemessen, erscheint die Frage nach einer ,objektiven Realität“" weniger relevant (Mattissek/Pfaffenbach/ Reuber 2013: 31). Beispielsweise in der konstruktivistischen Humangeographie rückt dann vielmehr die Analyse von ,raumbezogenen Images, Imaginations, Repräsentationen, Regionalisierungen, Leitbilder etc., die in einer Gesellschaft kursieren", in den Vordergrund (Mattissek/ Pfaffenbach/Reuber 2013: 33). Für Bryman (2004: 18) tritt an die Stelle von objektiver Realität die sich ständig ändernde subjektive Realität. Um auf eine Metaebene zu gelangen, untersuchen einige Forscher Diskurse und achten dabei besonders auf Sprache, die nicht als ein neutrales Medium verstanden wird, sondern als ein prägender Faktor für die Wahrnehmung der Welt.

Konstruktivistische Forschung ist anspruchsvoll und unterliegt selbst der Kritik. Dies soll am Beispiel des Aufsatzes von Jan Balke und Mario Reimer in „Raumforschung und Raumordnung“ gezeigt werden (Balke/Reimer 2016). Dazu ist jedoch zunächst auf zwei wichtige einschlägige, mit dem Konstruktivismus verbundene Begriffe einzugehen: Diskursanalyse und Gouvernementalität.

\section{Foucault: Diskursanalyse und Gouvernementalität}

Was die populären Begriffe Diskurs und Diskursanalyse bedeuten, wird sehr unterschiedlich definiert und eingeordnet; es gibt auch sehr verschiedene Ansätze der Diskursanaly- 
se (Flick 2007: 428 ff.; Schmidt 20081). Der sicherlich am häufigsten genannte Autor ist hier Michel Foucault. Er vertritt den poststrukturalistisch geprägten Ansatz, der sich intensiv mit Machtverhältnissen und Herrschaft auseinandersetzt (vgl. Foucault 1982; Foucault 1979). Auf ihn beziehen sich auch Balke und Reimer. Foucault ist sehr interpretationsbedürftig, weshalb sich der Autor hier auf Sekundärliteratur mit Einführungscharakter (insbesondere Mattissek/ Pfaffenbach/Reuber 2013 und Rosol/Schipper 2014) stützt. ${ }^{2}$

Der Kritische Rationalismus lässt, wenn man von den erwähnten Einschränkungen absieht, prinzipiell offen, welche Forschungsziele und Forschungsgegenstände gewählt werden. Im Gegensatz dazu verbindet sich bei der hier betrachteten Spielart der Diskursanalyse eine bestimmte konstruktivistische Position mit einem bestimmten Forschungsinteresse, nämlich der kritischen Analyse von Machtverhältnissen. Hier besteht eine Gemeinsamkeit mit der kritischen Theorie der Frankfurter Schule (z. B. Max Horkheimer), die sich vom marxistisch orientierten Ansatz her als emanzipatorisch versteht. Wissenschaft soll der Aufklärung dienen, sich aber nicht für eine schon vorhandene gesellschaftliche Realität instrumentalisieren lassen (Schülein/Reitze 2012: 134). In ähnlicher Weise versuchen die Diskursanalytiker Verhältnisse offenzulegen bzw. zu entlarven. Deshalb ist es keine Überraschung, dass sie sich stark mit dem Neoliberalismus und seinen sozialen Auswirkungen beschäftigen.

Es geht hier beim Diskursbegriff nicht um Debatten oder Diskussionen, obwohl sich auch unterschiedliche Diskurse gegenüberstehen können. Diskurs meint vielmehr ,überindividuelle Muster des Denkens, Sprechens, Sich-selbst Begreifens [sic!] und Handelns sowie Prozesse, in denen bestimmte Vorstellungen und Handlungslogiken hergestellt und immer wieder verändert werden" (Glasze/Mattissek 2009: 11 f.). Der Diskursbegriff geht damit weit über die Betrachtung von Argumentationen, Positionen oder Haltungen der Akteure hinaus, die auch etwa im akteurzentrierten Institutionalismus von Mayntz und Scharpf (Scharpf 1997) angelegt ist. Dabei ist anzumerken, dass bei der Diskursanalyse die Akteure und Institutionen nur eine sehr untergeordnete Rolle spielen.

Die Erkenntnis des Konstruktivismus, dass zumindest in der sozialen Welt nichts naturgegeben ist, gibt den Diskursanalytikern den Anlass, Dinge als konstruiert nachzuweisen, die vielen Menschen doch schon als naturgegeben erscheinen. Damit verbindet sich der Wunsch einer (Re-)Po-

\footnotetext{
1 Vgl. auch die Beiträge in Heft 1.2002 der Zeitschrift „European Political Science“"

${ }^{2}$ Foucault ist aus der Sicht des Autors sehr interpretationsbedürftig. Foucault destillierte seine nicht leicht zugänglichen Erkenntnisse etwa zu Macht, Wissen, Diskurs und Gouvernementalität z. B. aus der historischen Analyse gesellschaftlicher Phänomene wie Wahnsinn, Disziplinierung in Gefängnissen, Sexualität, Krieg und Herrschaft durch Fürsten bzw. Könige.
}

litisierung der betrachteten gesellschaftlichen Verhältnisse. Gesucht wird nach vielleicht auch verdeckten Konflikten sowie hegemonialen Diskursen, die Einzelne oder Gruppen marginalisieren. Den Zugang dazu, Dinge zu erkennen, die anderen verborgen bleiben, bietet die Sprache (vgl. ausführlich Mattissek/Pfaffenbach/Reuber 2013: 252 ff.).

Eng mit diskursanalytischen Ansätzen verknüpft ist die Gouvernementalitätsforschung, die ebenfalls auf Foucault zurückgeht. Sie beschäftigt sich mit Fragen der „Regierung“ von Individuen. Regierung wird dabei definiert als „die Gesamtheit der Institutionen und Praktiken, mittels derer man Menschen lenkt, von der Verwaltung bis zur Erziehung" (Foucault 1996: 118). Im Vordergrund steht die Untersuchung von vier zusammenhängenden, diskursiv geprägten Aspekten (Rosol/Schipper 2014), die in der Soziologie, der Politologie und sogar der Ökonomik nicht völlig fremd sind: Bei der Problematisierung geht es sehr vereinfacht darum, welche Phänomene in einer Gesellschaft zu einem Problem gemacht werden. Rationalitäten des Regierens sind die vorherrschenden Denk- und Handlungslogiken, auf die sich das Regierungshandeln gründet. Der Aspekt der Subjektivierung verweist darauf, dass sich Individuen mit ihrer Identität in Strukturen einpassen. Technologien der Regierung beinhalten Fremdtechnologien, bei denen der Staat beispielsweise Ge- und Verbote ausspricht. Besonders interessant sind die Selbsttechnologien, bei denen eine entsprechende Subjektivierung dafür sorgt, dass sich die Individuen von selbst systemkonform verhalten.

\section{Grenzen auch hier: Kritik an der Forschung zur Regionsbildung}

Während am Beispiel des Zurufs von Horst Zimmermann deutlich wurde, wie sehr alternative bzw. ergänzende Forschungsansätze erforderlich sind, macht das folgende Beispiel auf die Schwierigkeit der Anwendung des betrachteten konstruktivistisch-diskursanalytischen Ansatzes aufmerksam. Jan Balke und Mario Reimer befassen sich in ihrem Beitrag mit dem Thema „Regionsbildung im Schatten des Metropolendiskurses. Das Fallbeispiel der Region Südwestfalen“" (Balke/Reimer 2016), wobei sie dem beschriebenen Gouvernementalitätsansatz von Foucault folgen. Sie wenden diesen auf einen Kern der Raumforschung an: die Region und die Regionsbildung. Einen Hinweis auf den Einsatz besonderer sprachwissenschaftlicher Methoden gibt es nicht. Untersuchungsfall ist die Region Südwestfalen, in

\footnotetext{
${ }_{3}$ Die REGIONALEN sind ein Instrument der regionalisierten Strukturpolitik des Landes Nordrhein-Westfalen. Dem Vorbild der Internationalen Bauausstellung (IBA) Emscher Park folgend, wird die projektorientierte regionale Zusammenarbeit gefördert (vgl. http://www. regionalen.nrw.de; 03.03.2017).
} 
der 2013 eine REGIONALE ${ }^{3}$ durchgeführt wurde. Ziel der Studie von Balke und Reimer ist die Vertiefung des Verständnisses der Regionsbildung sowie die explorative Prüfung der Nutzbarkeit des konstruktivistischen Ansatzes. Die Autoren stellen zunächst das Konzept der Gouvernementalität dar und führen dann die Idee der „Soft Spaces“ als Ausdruck neoliberaler Gouvernementalität ein. Im empirischen Teil wird die REGIONALE in Nordrhein-Westfalen beschrieben. Auf die Analyse von Rationalitäten und Technologien der Regionsbildung folgt das Fazit.

Beeindruckend ist, wie mit der Nutzung des Begriffsapparates Foucaults eine Distanz zum Forschungsgegenstand hergestellt wird. In der Tat gelingt es Balke und Reimer, Dinge in einer Weise zu thematisieren, die neu, zumindest sehr ungewohnt ist. Mitunter scheint es, als kämen die Autoren aus einer anderen Welt, was innerhalb des Paradigmas sicherlich als Erfolg zu verstehen ist. Die Frage ist allerdings, welche neuen, gut fundierten Erkenntnisse sich daraus ableiten lassen. Dies soll an einem Beispiel diskutiert werden.

Als eine von drei Rationalitäten der Regionsbildung wird die „Notwendigkeit interkommunaler Kommunikation“ (Balke/Reimer 2016: 300) genannt. Die Autoren schreiben, dass ,die interkommunale Kooperation hier zum strukturpolitischen ,Allheilmittel ' zur endogenen Überwindung regionaler Herausforderungen erhoben wird" (Balke/ Reimer 2016: 300). Sie bezeichnen interkommunale Kooperation darüber hinaus als ,unhinterfragte Handlungslogik“ (Balke/Reimer 2016: 300). In beiden Fällen ist nicht klar, woraus sich das genau herleitet. In Anbetracht dessen, dass das Land Nordrhein-Westfalen schon seit 1987 Erfahrungen mit der Regionalisierung gesammelt hat und es eine Fülle von auch kritischer Literatur dazu gibt, hätte man sich eine genauere Begründung gewünscht. Vielleicht wäre es auch schon hilfreich gewesen, deutlich zu machen, welche vorhandenen alternativen Handlungslogiken bzw. Gegendiskurse die interkommunale Kooperation als Rationalität in Frage stellen könnten. Unklar bleibt auch, wie sich diese Aussagen zu den konkreten Ergebnissen aus der bisherigen Literatur verhalten. Danielzyk, Kemming und Reimer (2011), beispielsweise, kommen zu einer durchaus positiven Einschätzung des Instruments der REGIONALEN.

Was die Analyse von Konflikten anbelangt, stellen die Autoren fest, dass es solche zwischen den Kommunen untereinander und zwischen den verschiedenen politischadministrativen Ebenen gibt (,konfliktive Machtbeziehungen“, Balke/Reimer 2016: 300). Die Autoren scheinen hier aber kaum etwas aufzudecken, was vorher unbekannt oder unbeachtet geblieben wäre. Sie folgern unter anderem, dass „seitens der Landespolitik eine regionale Responsibilisierung des Erfolgs interkommunaler Kooperation" (Balke/ Reimer 2016: 300) erfolgt. Viele Leser dürften sich fragen, was damit genau gesagt werden sollte. Eine normative Einordnung wurde explizit nicht vorgenommen (Balke/Reimer 2016: 304), wobei jedoch die verwendeten Begriffe eher kritisch klingen.

Der Beitrag von Balke und Reimer scheint insgesamt gesehen zu zeigen, dass es nicht einfach ist, den Foucaultschen Ansatz zur Analyse des hier gewählten Gegenstands mit deutlichem Gewinn anzuwenden. Die Herangehensweise wirkt letztlich etwas aufgesetzt. Gerade bei einem explorativen Versuch der Anwendung des Ansatzes wäre eine kritische Reflektion erhellend gewesen. Das beinhaltet erstens die Frage, welche Aussagen sich überzeugend aus dem empirischen Material ableiten lassen. Zweitens hätte angesprochen werden können, wie sich diese Aussagen zu den konkreten bereits vorliegenden empirischen Ergebnissen verhalten. Schließlich wäre es interessant gewesen, etwas allgemeiner die Leistungsfähigkeit des Foucaultschen Ansatzes mit der eher staatstheoretischen regionalen $\mathrm{Go}^{-}$ vernance-Forschung $\mathrm{zu}$ vergleichen, von der sich die $\mathrm{Au}$ toren zu Beginn des Aufsatzes abgrenzen (Balke/Reimer 2016: 294, 296). Letztere wurde wesentlich von Dietrich Fürst (z. B. Fürst 1999; Fürst/Lahner/Pollermann 2005 und Fürst 2010) und damit letztlich auch vom akteurzentrierten Institutionalismus (Scharpf 1997) geprägt. Vielleicht ist die hier vorgetragene Kritik paradigmenfremd. Möglicherweise sind aber auch die Autoren nicht exakt dem selbst gewählten Paradigma gefolgt.

\section{Für ein besseres Verständnis}

Aus den vorangegangenen Überlegungen können die folgenden Schlussfolgerungen zum machtkritischen konstruktivistisch-diskurstheoretischen Paradigma bzw. zur Foucaultschen Gouvernementalitätsanalyse gezogen werden. Der Ansatz hat seine Potenziale und ist aus der Sozialwissenschaft nicht wegzudenken. Man sollte ihm nicht ohne Verständnis begegnen, wie etwa Horst Zimmermann (2012) dies in seinem Diskussionsbeitrag in „Raumforschung und Raumordnung" tat. Der Ansatz folgt nicht zwingend aus der Erkenntnis, dass letztlich alles auf irgendeine Weise konstruiert ist, definiert sich aber über die Annahme der Konstruiertheit. Es gilt auch, dass machtkritische Untersuchungen nicht auf die Foucaultsche Herangehensweise angewiesen sind. Um das Spektrum vorhandener Ansätze zur Analyse von regionaler Governance in der Fürst'schen Tradition weiterzuentwickeln, ist es nicht zuletzt möglich, den akteurzentrierten Institutionalismus durch Ansätze der Diskursanalyse zu erweitern, die nicht auf Foucault rekurrieren. So verbindet etwa Lintz (2016) den akteurzentrierten Institutionalismus mit dem diskursiven Institutionalismus (vgl. Schmidt 2008). 
Aus der Betrachtung des Aufsatzes von Jan Balke und Mario Reimer (2016) ergibt sich die Frage, inwiefern sich die vorgenommene Analyse mit früheren, insbesondere auf einer anderen methodologischen Grundlage gewonnenen Erkenntnissen aus der Forschung beschäftigen müsste. Vielleicht könnte ja paradigmentreu zumindest die entsprechende Literatur als Teil der Diskurse betrachtet werden und somit in die Analyse einfließen. Obwohl es dazu schon Beiträge gibt (z. B. Gailing/Leibenath 2015), ist auch nicht allgemein klar, unter welchen Bedingungen bzw. bei welchen Forschungsgegenständen der anspruchsvolle Foucaultsche Ansatz seine Potenziale voll entfaltet. Es besteht die Gefahr, dass dessen Anwendung unter Umständen etwas aufgesetzt wirkt.

Angesichts der herausfordernden theoretischen Grundlagen, den häufig gewählten Forschungsfragen und der $\mathrm{Zu}$ rückhaltung beim Geben von Politikempfehlungen dürfte besonders unter Praktikern die Befürchtung bestehen, dass sich die konstruktivistisch-diskursanalytische Wissenschaft zu weit von den gesellschaftlichen Problemen entfernt und für die alltägliche Planungspraxis nicht mehr relevant ist. Ersteres dürfte keinesfalls generell zutreffen, da verteilungspolitische Fragestellungen von größter Aktualität sind, wobei allerdings die Perspektive von Macht- und Herrschaftsverhältnissen in der deutschen Raumforschung bislang eher selten eingenommen wurde. Relevant ist der Ansatz auch für die alltägliche Planungspraxis, allerdings in einem völlig anderen Sinne. Es geht, wie erwähnt, weniger darum, instrumentelles Wissen für die bestehende Praxis anzubieten, sondern eher darum, die bestehende Praxis als solche kritisch zu hinterfragen.

Jede Wahl eines Forschungsgegenstandes oder eines methodologischen Ansatzes ist mit einer unvermeidbaren Wertentscheidung verbunden. In der Tendenz scheint es, dass Vertreter der machtkritischen Diskursanalyse und Gouvernementalitätsforschung häufig von einem Ideal sehr starker Gleichheit von Menschen ausgehen und schneller und grundsätzlicher institutionelle Gegebenheiten in Frage stellen. Insofern ist der Ansatz auch im Vergleich zur sonstigen Forschung eher radikal in seiner Orientierung. Das Weltbild ist eher macht- und konflikt- als konsensbetont. Vielleicht spiegeln sich darin reale Entwicklungen in der Gesellschaft wider. Möglicherweise trägt der Ansatz auch zu einer größeren Betonung von Konflikten in der Gesellschaft bei.

Insgesamt gesehen steigen im Zuge der zunehmenden Globalisierung der deutschen Raumwissenschaft die methodologischen und theoretischen Ansprüche an die Forschung, und die Landschaft der entsprechenden Zugänge wird unübersichtlicher. Dies kann zu Spannungen sowohl zwischen wissenschaftlichen Lagern als auch zwischen Wissenschaft und Praxis führen.
Gerade in Deutschland, wo es unter anderem durch die Akademie für Raumforschung und Landesplanung (ARL) eine lange Tradition zur Verbindung von Wissenschaft und Praxis in den Raumwissenschaften gibt, sollte versucht werden, die erwähnten Spannungen fruchtbar zu machen. Auch wenn kein Konsens zu erwarten ist, wäre es wichtig, zumindest das allgemeine Verständnis für die Gemeinsamkeiten und Unterschiede der verschiedenen möglichen Ansätze im Dickicht von Wissenschaftstheorie und inhaltlicher Schwerpunktsetzung zu verbessern. Es muss in einer offenen Diskussion Forschern, Praktikern und Studenten deutlicher werden, was es bedeutet, wenn ein bestimmter Ansatz angewandt wird. Hilfreich wäre daher unter anderem eine raumwissenschaftlich orientierte Publikation zu methodologischen Fragen, die gemeinsam von Vertretern der verschiedenen Ansätze erarbeitet wird. $\mathrm{Zu}$ achten wäre unbedingt darauf, dass das große ,mittlere Feld' der Forschungspraxis genügend Beachtung findet, das weder vom Kritischen Rationalismus noch vom konstruktivistisch-diskursanalytischen Ansatz abgedeckt wird.

Danksagung Der Autor bedankt sich bei Markus Leibenath und Holger Leimbrock für wertvolle Kommentare zum Manuskript und für teils kontroverse, aber immer spannende Diskussionen. Dank gebührt ebenfalls den anonymen Gutachtern für zahlreiche hilfreiche Hinweise.

\section{Literatur}

Balke, J.; Reimer, M. (2016): Regionsbildung im Schatten des Metropolendiskurses. Das Fallbeispiel der Region Südwestfalen. In: Raumforschung und Raumordnung 74, 4, 293-305.

Bryman, A. (2004): Social Research Methods. Oxford.

Danielzyk, R.; Kemming, H.; Reimer, M. (2011): Die REGIONALEN in NRW - Impulse der IBA Emscher Park. In: Reicher, C.; Niemann, L.; Uttke, A. (Hrsg.): Internationale Bauausstellung Emscher Park: Impulse. Essen, 276-284.

Flick, U. (2007): Qualitative Sozialforschung. Eine Einführung. Reinbek.

Foucault, M. (1979): Governmentality. In: Ideology and Consciousness $6,1,5-21$

Foucault, M. (1982): The subject and power. In: Critical Inquiry 8, 4, $777-795$.

Foucault, M. (1996): Der Mensch ist ein Erfahrungstier. Gespräch mit Ducio Trombadori. Frankfurt am Main.

Fürst, D. (1999): Auswertung von Erfahrungen zur Kooperation in Regionen. In: Raumforschung und Raumordnung 57, 1, 53-58.

Fürst, D. (2010): Regional Governance. In: Benz, A.; Dose, N. (Hrsg.): Governance - Regieren in komplexen Regelsystemen. Eine Einführung. Wiesbaden, 49-68.

Fürst, D.; Lahner, M.; Pollermann, K. (2005): Regional Governance bei Gemeinschaftsgütern des Ressourcenschutzes: das Beispiel Biosphärenreservate. In: Raumforschung und Raumordnung 63, 5, 330-339.

Gailing, L.; Leibenath, M. (2015): The social construction of landscapes: Two theoretical lenses and their empirical applications. In: Landscape Research 40, 2, 123-138.

Glasze, G.; Mattissek, A. (2009): Diskursforschung in der Humangeographie: Konzeptionelle Grundlagen und empirische Operationalisierungen. In: Glasze, G.; Mattissek, A. (Hrsg.): Handbuch Diskurs und Raum. Theorien und Methoden für die Humangeo- 
graphie sowie die sozial- und kulturwissenschaftliche Raumforschung. Bielefeld, 11-59.

Kilper, H.; Heiland, S.; Leibenath, M.; Tzschaschel, S. (2012): Die gesellschaftliche Konstituierung von Kulturlandschaft. In: Raumforschung und Raumordnung 70, 2, 91-94.

Lintz, G. (2016): A conceptual framework for analysing inter-municipal cooperation on the environment. In: Regional Studies 50, 6, 956-970.

Mattissek, A.; Pfaffenbach, C.; Reuber, P. (2013): Methoden der empirischen Humangeographie. Braunschweig.

Popper, K. R. (1984) [1972]: Objektive Erkenntnis. Ein evolutionärer Entwurf. Deutsche Fassung der 4. verbesserten und ergänzten englischsprachigen Ausgabe. Hamburg.

Popper, K. R. (1994) [1935]: Logik der Forschung. Tübingen.

Prim, R.; Tilmann, H. (2000): Grundlagen einer kritisch-rationalen Sozialwissenschaft. Studienbuch zur Wissenschaftstheorie Karl R. Poppers. Wiebelsheim.
Rosol, M.; Schipper, S. (2014): Das Foucaultsche Konzept der Gouvernementalität. In: Oßenbrügge, J.; Vogelpohl, A. (Hrsg.): Theorien in der Raum- und Stadtforschung. Einführungen. Münster, 271289.

Sander, W. (2014): Wissenschaftstheoretische Grundlagen der politischen Bildung: Konstruktivismus. In: Sander, W. (Hrsg.): Handbuch politische Bildung. Bonn, 77-89. = Schriftenreihe der Bundeszentrale für politische Bildung 1420.

Scharpf, F. W. (1997): Games Real Actors Play. Actor-Centered Institutionalism in Policy Research. Boulder/Colorado.

Schmidt, V. A. (2008): Discursive Institutionalism: The Explanatory Power of Ideas and Discourse. In: Annual Review of Political Science 11, 303-326.

Schülein, J. A.; Reitze, S. (2012): Wissenschaftstheorie für Einsteiger. Wien.

Zimmermann, H. (2012): Zuruf: Landschaft aus ökonomischer Sicht. In: Raumforschung und Raumordnung 70, 6, 481-483. 